\title{
Monitoramento e armazenamento de dados de temperatura e luminosidade utilizando redes de sensores ZigBee
}

\author{
Monitoring and storing of temperature and luminosity data using ZigBee sensor networks \\ Monitoreo y almacenamiento de datos de temperatura y luminosidad usando redes de sensores \\ ZigBee
}

Recebido: 19/08/2021 | Revisado: 25/08/2021 | Aceito: 26/08/2021 | Publicado: 29/08/2021

\author{
Gilberto de Melo Junior \\ ORCID: https://orcid.org/0000-0002-2317-7779 \\ Instituto Federal de Educação, Ciência e Tecnologia do Pará, Brasil \\ E-mail: gilberto.melo@ifpa.edu.br
}

\begin{abstract}
Resumo
O artigo proposto relata o desenvolvimento de uma rede de sensores sem fio baseado no protocolo ZigBee. Desenvolveu-se um dispositivo Coordenador e dispositivos finais para atuar na rede, realizando monitoramento de dados de luminosidade e temperatura de um ambiente. A rede foi testada e validada através do monitoramento dos dados pertinentes, que por sua vez foram apresentados em um servidor web, o EmonCMS, e gravados em um cartão de memória Secure Digital (SD). O projeto desenvolvido apresentou baixo consumo energético.
\end{abstract}

Palavras-chave: Redes de sensores; ZigBee; Temperatura; Luminosidade.

\begin{abstract}
The proposed article reports the development of a wireless sensor network based on the ZigBee protocol. A Coordinating device and a final device were developed to act on the network, monitoring luminosity and temperature data in an environment. The network was tested and validated by monitoring the relevant data, which in turn was presented on a web server, EmonCMS, and recorded on a Secure Digital (SD) memory card. The developed project had low energy consumption.
\end{abstract}

Keywords: Sensor networks; ZigBee; Temperature; Luminosity.

\section{Resumen}

El artículo propuesto informa del desarrollo de una red de sensores inalámbricos basada en el protocolo ZigBee. Se desarrolló un Dispositivo Coordinador y un Dispositivo final para actuar en la red, monitoreando los datos de luminosidad y temperatura en un ambiente. La red fue probada y validada monitoreando los datos relevantes, que a su vez fueron presentados en un servidor web, EmonCMS, y registrados en una tarjeta de memoria Secure Digital (SD). El proyecto desarrollado tuvo un bajo consumo energético.

Palabras clave: Redes de sensores; ZigBee; Temperatura; Luminosidad.

\section{Introdução}

A automação residencial e comercial utilizando comunicação de redes sem fio tem se tornado uma tecnologia muito promissora devido às facilidades na instalação, mobilidade e flexibilidade. As tecnologias de monitoramento têm crescido exponencialmente nos últimos anos. Cada vez mais, as pessoas necessitam estar conectadas a tudo o que acontece na residência, no trabalho e nos demais ambientes onde frequentam.

A tecnologia ZigBee envolve protocolo de redes de sensores sem fio e vem sendo utilizada em diversas áreas. No trabalho de De Sousa et al. (2008), propuseram o desenvolvimento de protótipo de hardware e de software para monitorar, controlar e parametrizar máquinas lavadoras de roupas numa rede sem fio ZigBee através de uma interface virtual remota.

Tanto no trabalho de Pechoto et al. (2012) e Stuhler et al. (2012) desenvolveram-se uma rede de sensores para monitoramento em tempo real de enchentes de rios urbanos e enchentes. Avelino et al. (2013) desenvolveram um sistema de monitoramento em tempo real de ativos hospitalares, onde era monitorado temperaturas de ambientes que armazenavam remédios. Sabo et al. (2011) desenvolveram uma rede de sensores para um sistema meteorológico para fins agrícolas. 
Tavoloni Jr et al. (2013) analisaram a mineração de dados em aplicações domésticas Smart Grid (SG), com coleta de dados efetuadas por meio de redes de sensores sem fio Zigbee. No estudo de Moyolema et al. (2020) foi apresentada uma metodologia baseada nas técnicas de Otimização por Enxame de Partículas (PSO) e de Agrupamento por Grafos (AG) para determinar os locais para instalação dos Concentradores Inteligentes Sem Fio (CISF) e agrupar os Medidores Sem Fio Inteligentes (MISF), ambos empregando tecnologias de comunicação Zigbee, para monitoramento de redes de baixa tensão com geração distribuída.

O trabalho de Oliveira (2015) analisou a viabilidade de utilização do protocolo ZigBee em aplicações de Rede Dinâmica de Sensores sem fio (RDSSF). Já no trabalho de Tose et al. (2012) desenvolveram uma rede de sensores sem fio, de pequeno porte, adaptada para monitoramento de equipamentos eletromecânicos e condições ambientais de uma Estação de Tratamento de Esgoto (ETE).

Silva et al. (2017), Sírio (2018) e de Melo Junior (2020b) utilizaram o padrão ZigBee no desenvolvimento de uma rede de sensores sem fio na topologia Mesh (malha).

Tais projetos descritos evidenciam a importância do estudo de tecnologias de redes de sensores sem fio, no qual o protocolo ZigBee se destaca pelo baixo consumo de energia elétrica e pela flexibilidade na instalação da rede.

Este projeto tem o intuito de utilizar redes de sensores sem fio, com tecnologia ZigBee, para monitoramento de dados de temperatura e luminosidade, em ambientes residenciais e comercias. Na Seção 2 será apresentado os materiais e métodos; seção 3 apresentará os resultados obtidos; seção 4 apresenta conclusões.

\section{Materiais e Métodos}

O propósito do projeto foi desenvolver um sistema de rede de sensores sem fio para monitoramento de ambientes residenciais ou comerciais, armazenando dados de temperatura e luminosidade. O desenvolvimento iniciou-se com a criação de dispositivos de rede, contendo módulos XBee, que utilizam o protocolo de comunicação ZigBee, como meio de transmissão.

Os métodos utilizados para apresentação do resultado da metodologia empregada no estudo de caso, são métodos qualitativos, onde os pesquisadores exploraram o funcionamento da rede de sensores sem fio e também analisaram o consumo de energia dessa rede (Pereira et al., 2018).

O ZigBee é um protocolo de comunicação para redes sem fio de curto alcance e de baixa velocidade de transmissão de dados (Farahani, 2011). As suas bases foram estabelecidas no protocolo Home RFLite criado pela Philips. A tecnologia foi pela primeira vez apresentada ao público com o nome de ZigBee em julho de 2005 (Saleiro e Ey, 2009).

O ZigBee é implementado sobre o padrão IEEE 802.15.4 desenvolvido pelo Instituto de Engenheiros Eletricistas e Eletrônicos (IEEE), o qual define as camadas física e de enlace da rede. De acordo com Da Silva (2007), o ZigBee possui determinadas características que o tornam absolutamente distinto dos demais:

- Baixo consumo de energia;

- Pilha protocolar de implementação simplificada (código de tamanho menor de um quarto da pilha do Bluetooth), conduzindo a interfaces de baixo custo;

- Possibilidade de suportar uma elevada densidade de nós por rede (máximo de 65535) dispositivos por cada ZigBee coordenador;

- Admite diferentes topologias de rede: estrela, malha ou grupo de árvores.

O ZigBee distingue em sua rede três tipos de dispositivos lógicos: Coordenador, Roteador e Final. Neste projeto foi desenvolvido um Coordenador e três dispositivos Final. A rede utilizando ZigBee suporta apenas um Coordenador cuja função 
é formar a rede e atribuir endereços. Os dispositivos Final consomem pouca energia, devido ao modo Sleep integrado. Eles são responsáveis pela ação de controle ou monitoramento associado a sensores e atuadores.

O XBee é um módulo de radiofrequência desenvolvidos pela Digi International, chamados também de Digi XBee. Os primeiros módulos XBee foram desenvolvidos em 2005, e são baseados no padrão IEEE 802.15.4, utilizando o ZigBee como protocolo de comunicação. Inicialmente foram desenvolvidos dois modelos: XBee e XBee-PRO. Os módulos podem assumir vários tipos de topologia com o mínimo de conexões possíveis e com baixo consumo de energia na comunicação (Manual, 2008).

$\mathrm{O}$ estudo de caso deste projeto, constituiu no monitoramento e armazenamento de dados de luminosidade e temperatura em três ambientes utilizando um dispositivo Coordenador e por dispositivos Final, nos quais foram nomeados de dispositivos Modelo 1. O mesmo consistiu no monitoramento e armazenamento de dados de luminosidade e temperatura em três ambientes. Os dispositivos Modelo1 foram responsáveis por fazerem a leitura dos sensores e enviar os dados para o coordenador, que realizou o processamento e o armazenamento dos dados. O diagrama deste estudo é apresentado na Figura 1.

Figura 1. Diagrama do estudo de caso de monitoramento de dados desenvolvido.

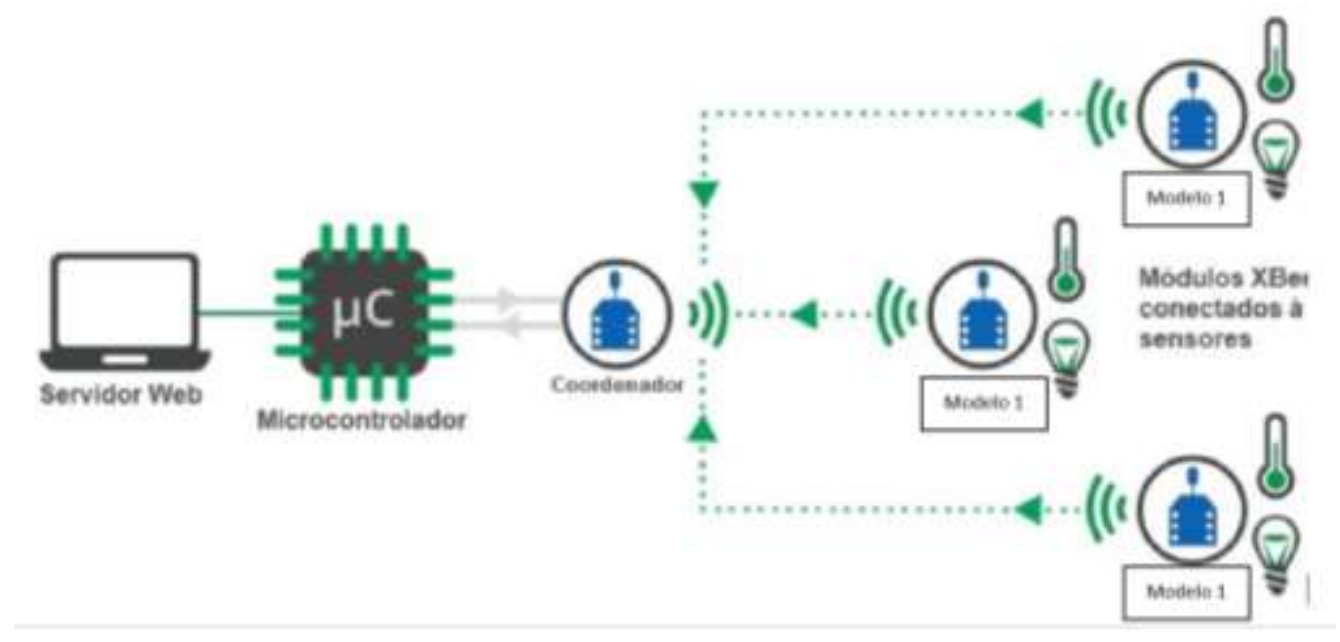

Fonte: Autores.

O dispositivo Coordenador foi composto por um Arduino Mega 2560, uma Ethernet Shield W500 para Arduino, uma XBee Shield para Arduino, um XBee-PRO S2 e um Relógio de Tempo Real (RTC - Real Time Clock) DS1307, sendo responsável por receber os dados dos dispositivos Modelo 1, processá-los e armazená-los, tanto em um cartão de memória quanto em um servidor web. Figura 2 ilustra o dispositivo Coordenador desenvolvido.

O dispositivo Modelo 1, foi desenvolvido integrando um XBee-PRO S2, um XBee Explorer Adapter, um sensor de luminosidade LDR e um sensor de temperatura LM35. De acordo com Instruments (1999) as séries LM35 são circuitos integrados precisos para medição de temperatura, com uma saída de tensão linearmente proporcional à temperatura em graus Celsius em um range de $-55,0^{\circ} \mathrm{C}$ à $150,0{ }^{\circ} \mathrm{C}$ e precisão de $\pm 3 / 4{ }^{\circ} \mathrm{C}$. Para o sensor de luminosidade, foi utilizado um dispositivo cuja resistência elétrica varia de acordo com a intensidade luminosa, também chamado de Resistor Dependente de Luz (LDR Light Dependent Resistor). Segundo Sunrom Technologies (2008), este componente apresenta uma resistência na faixa de 1,0 $\mathrm{M} \Omega$ à 0 Lux, diminuindo enquanto maior for a incidência de luz sobre o dispositivo. Figura 3 ilustra o dispositivo Modelo 1 desenvolvido.

O servidor web utilizado foi o EmonCMS, que é um aplicativo Web open-source para processamento, armazenamento e visualização de dados de temperatura, luminosidade, energia, entre outras grandezas. Como a maioria das aplicações web 
atuais, a arquitetura do EmonCMS utiliza-se de código Hyper Text Markup Language (HTML), Cascading Style Sheets (CSS) e Javascript para compor sua interface com o usuário.

Figura 2. Hardware utilizado como dispositivo Coordenador, onde em (1) são os conectores entre XBee Shield e o Arduino.

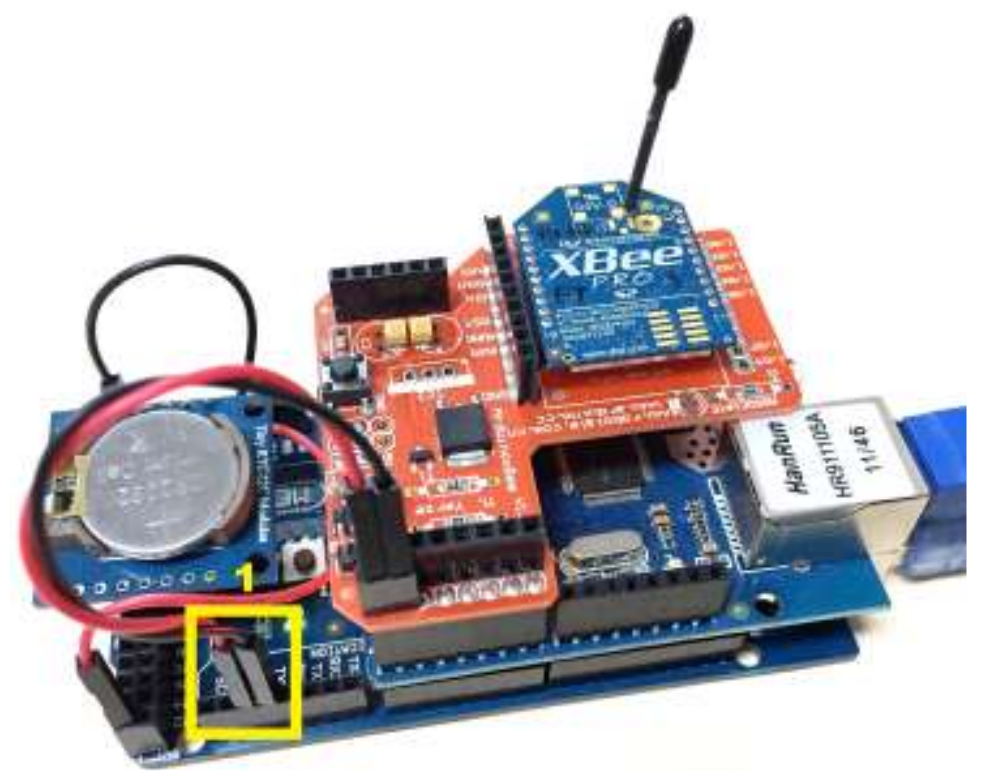

Fonte: De Melo Junior et al. (2020a).

Figura 3. Dispositivo Modelo 1 para monitoramento de temperatura e luminosidade.

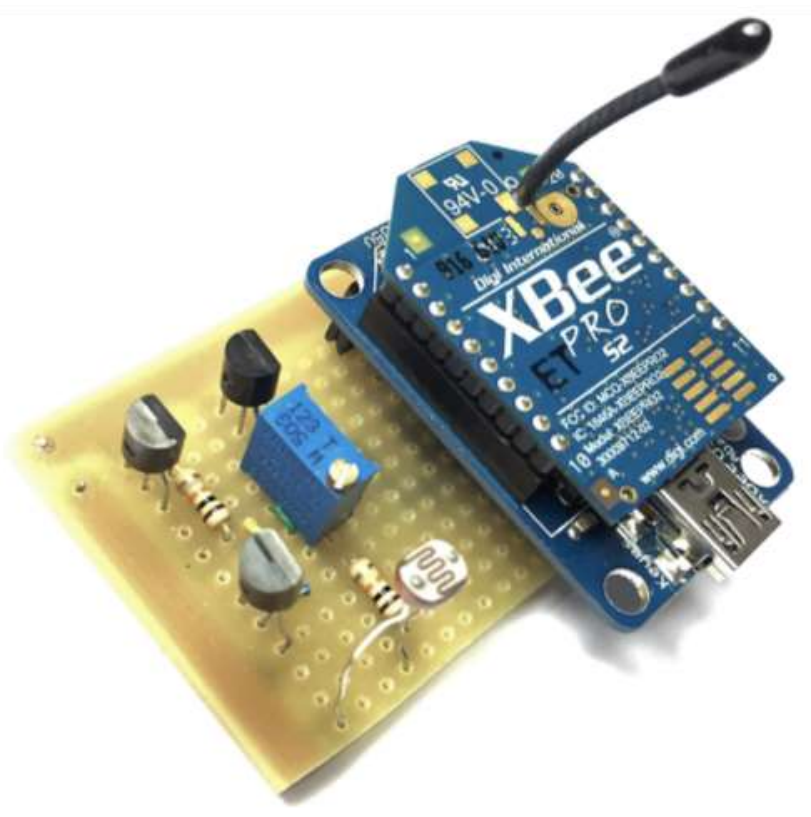

Fonte: De Melo Junior et al. (2020a).

O objetivo foi validar dos dispositivos Modelo 1 desenvolvidos, verificando a capacidade de leitura dos sensores e transmissão dos mesmos, além de analisar o processamento e armazenamento de dados feito pelo Coordenador.

Neste estudo, os módulos XBee dos dispositivos Modelo 1 entraram em modo Sleep (modo onde o módulo XBee consome pouquíssima energia) por um período e, ao entrarem no modo ativo, fizeram uma leitura dos sensores e transmitiram estes dados para o módulo coordenador. O módulo coordenador, por sua vez, recebeu os dados através do XBee e a placa Arduino os processou separando as informações de acordo com o módulo que as enviaram. Posteriormente, os dados foram 
armazenados no cartão SD e enviados ao EmonCMS. Além disso, houve a verificação do consumo de energia dos módulos em operação.

Este monitoramento foi realizado no Instituto Federal de Educação, Ciência e Tecnologia de Goiás, campus Goiânia, no laboratório NExT na sala T-309, a qual é composta por diferentes ambientes que possibilitaram a realização do estudo proposto.

\section{Resultados}

No estudo de caso foi realizado o monitoramento de temperatura e luminosidade de três ambientes diferentes. Este monitoramento foi feito utilizando dispositivos Modelo 1. O módulo XBee de cada ambiente foi programado para entrar em modo sleep por 5,0 minutos e permanecer em modo ativo por apenas 2,0 segundos. Durante o período ativo, os módulos fizeram uma leitura das portas $I / O$ analógicas e digitais e então enviaram os dados para o dispositivo Coordenador. Os dados chegaram ao coordenador em frames, na forma apresentada na Figura 4:

Figura 4. Exemplo de Frame que foram transmitidos para o dispositivo Coordenador.

\begin{tabular}{|l|l|l|l|l|l|l|l|l|l|l|l|l|l|l|l|l|l|l|l|l|l|l|l|}
\hline $7 \mathrm{E}$ & 00 & 16 & 92 & 00 & 13 & $\mathrm{~A} 2$ & 00 & 40 & $\mathrm{~A} 6$ & $\mathrm{FA}$ & $9 \mathrm{~A}$ & 00 & 04 & 01 & 01 & 00 & 00 & 05 & 00 & $\mathrm{~F} 0$ & 02 & $0 \mathrm{C}$ & $\mathrm{E} 1$ \\
\hline
\end{tabular}

Fonte: Autores.

As informações contidas neste frame foram:

- Byte inicial (Start delimiter): 7E;

- Tamanho do frame (Length): 0016 (20 bytes - contando a partir do endereço de origem);

- Tipo de frame (Frame type): 92 (92 - frame de dados);

- Endereço de origem (64-bit source address): 0013 A2 0040 A6 FA 9A;

- Endereço da rede de origem (16-bit source address): 00 04;

- Opções de recebimento (Receive options): 01;

- Número de amostras (Number of samples): 01;

- Estado das portas digitais (Digital channel mask): 0000 (0000 0000 - todas as portas digitais estão desabilitadas);

- Estado das portas analógicas (Analog channel mask): 05 (0000 0101 - portas A0 e A2 habilitadas);

- Valor da porta analógica AD0 (DIO0/AD0 Analog value): 00 F0 (281,53 mV);

- Valor da porta analógica AD2 (DIO2/AD2 Analog value): 02 0C (614,66 mV);

- Checksum: E1;

O dispositivo Coordenador, por meio da placa Arduino, foi responsável pelo processamento dos frames enviados pelos dispositivos Modelo 1. Inicialmente o microprocessador identificou o tipo de frame. Caso este fosse um frame de dados, seriam enviadas as informações do endereço do módulo remetente e os valores das portas analógicas. Com base no endereço de origem, o microprocessador armazenou os dados em suas respectivas variáveis e os enviou para o cartão SD e para o servidor web EmonCMS.

Caso o Arduino estivesse conectado ao computador, o processamento dos dados podia ser observado via Serial Monitor (comunicação serial do software Arduino), conforme ilustrado na Figura 5. 
Figura 5. Monitoramento do processamento de dados utilizando o Serial Monitor do software Arduino.

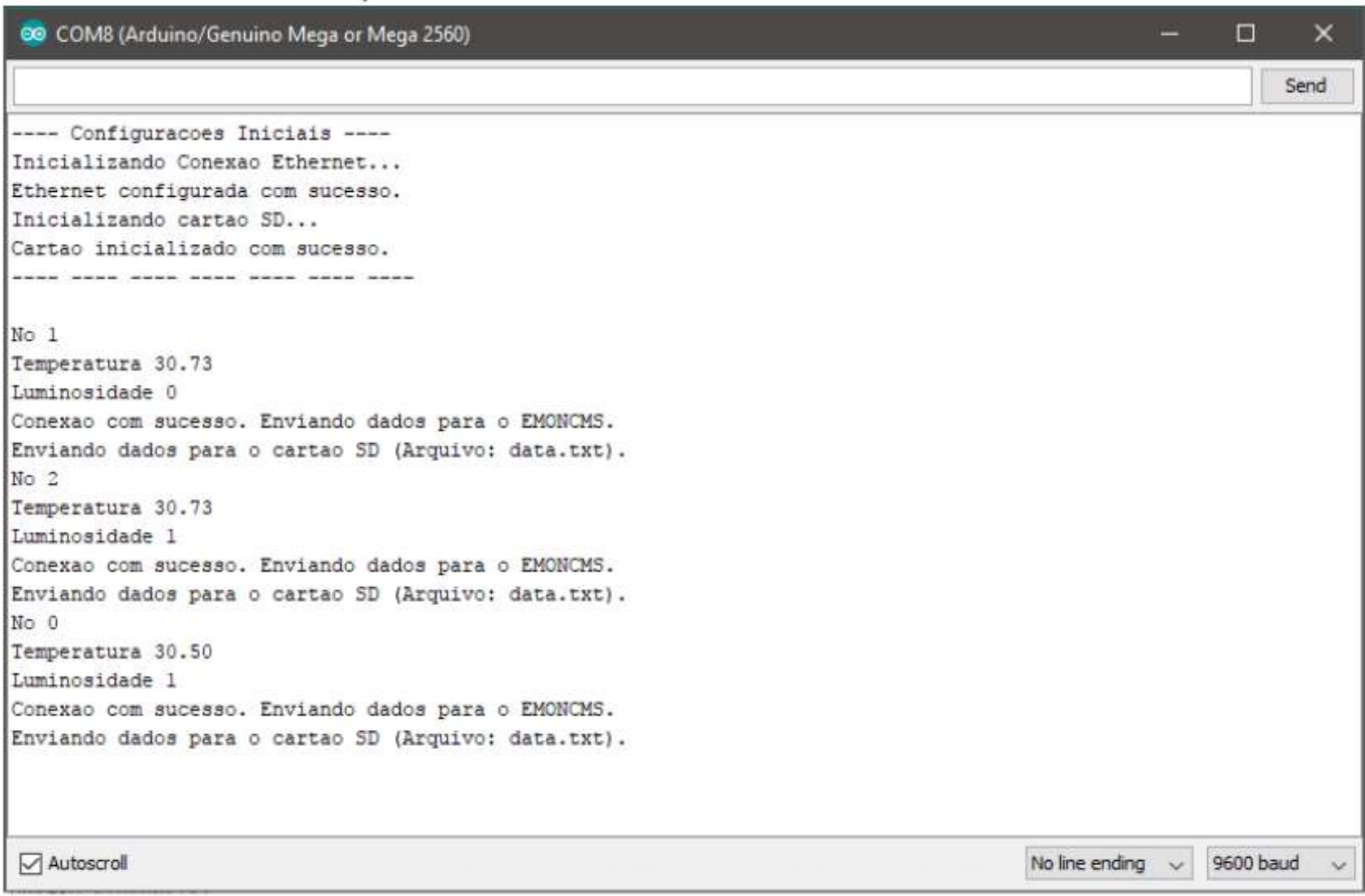

Fonte: Autores.

A Figura 6 apresenta como os dados de luminosidade e temperatura foram armazenados no cartão de memória SD, no qual o módulo RTC foi o responsável em fornecer as informações de data e hora.

Por fim, as informações foram enviadas para o servidos web EmonCMS. Para este estudo de caso foram criados Dashboards, no qual o primeiro deles foi desenvolvido para monitorar em conjunto dos três dispositivos Modelo 1, como ilustrado na Figura 7. Os demais foram criados para a visualização independente dos dados de cada um dos módulos, conforme apresentado na Figura 8.

Neste estudo de caso também foram realizadas análises de consumo de energia dos dispositivos Modelo 1. Foram utilizados transistores para chaveamento da alimentação dos sensores, fazendo com que os mesmos não consumissem energia durante o estado inativo dos módulos. Portanto, o dispositivo Modelo 1 foi alimentado com uma fonte de 5,0 V e foram realizadas medições do consumo de corrente durante os períodos ativos e inativos (modo sleep) do módulo. O Quadro 1 apresenta os resultados obtidos. 
Figura 6. Armazenamento de dados no cartão SD.

File Edit Format View Help

27.09.17, 06:00:39, No: 0 , Temperatura: 28.97, Luminosidade: 1

27.09.17, 06:01:59, No: 2, Temperatura: 27.92, Luminosidade: 0

27.09.17, 06:03:17, No: 1, Temperatura: 27.68, Luminosidade: 0

27.09.17, 06:07:33, No: 0, Temperatura: 29.21, Luminosidade: 1

27.09.17, 06:08:51, No: 2, Temperatura: 27.68, Luminosidade: 0

27.09.17, 06:10:09, No: 1, Temperatura: 27.57, Luminosidade: 0

27.09.17, 06:14:27, No: 0, Temperatura: 29.21, Luminosidade: 1

27.09.17, 06:15:43, No: 2, Temperatura: 27.92, Luminosidade: 0

27.09.17, $06: 17: 02$, No: 1, Temperatura: 27.57, Luminosidade: 0

27.09.17, $06: 21: 21$, No: 0 , Temperatura: 29.21, Luminosidade: 1

27.09.17, 06:22:35, No: 2, Temperatura: 27.92, Luminosidade: 0

27.09.17, $06: 23: 54$, No: 1, Temperatura: 27.57, Luminosidade: 0

27.09.17, 06:28:16, No: 0, Temperatura: 29.21, Luminosidade: 1

27.09.17, 06:29:27, No: 2, Temperatura: 27.92, Luminosidade: 0

27.09.17, 06:30:46, No: 1, Temperatura: 27.57, Luminosidade: 0

Fonte: Autores.

Figura 7. Dashboard para monitoramento dos três dispositivos Modelo 1.

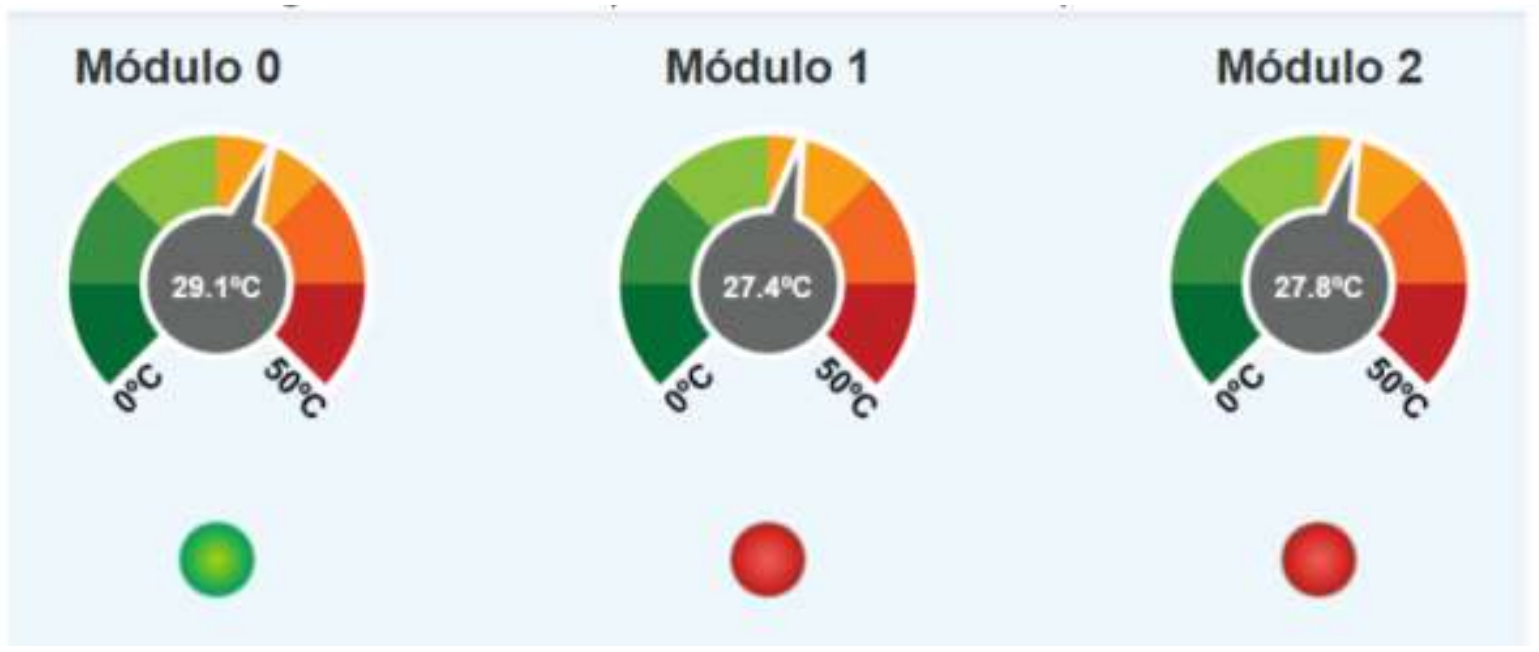

Fonte: Autores. 
Figura 8. Dashboard para monitoramento do ambiente.

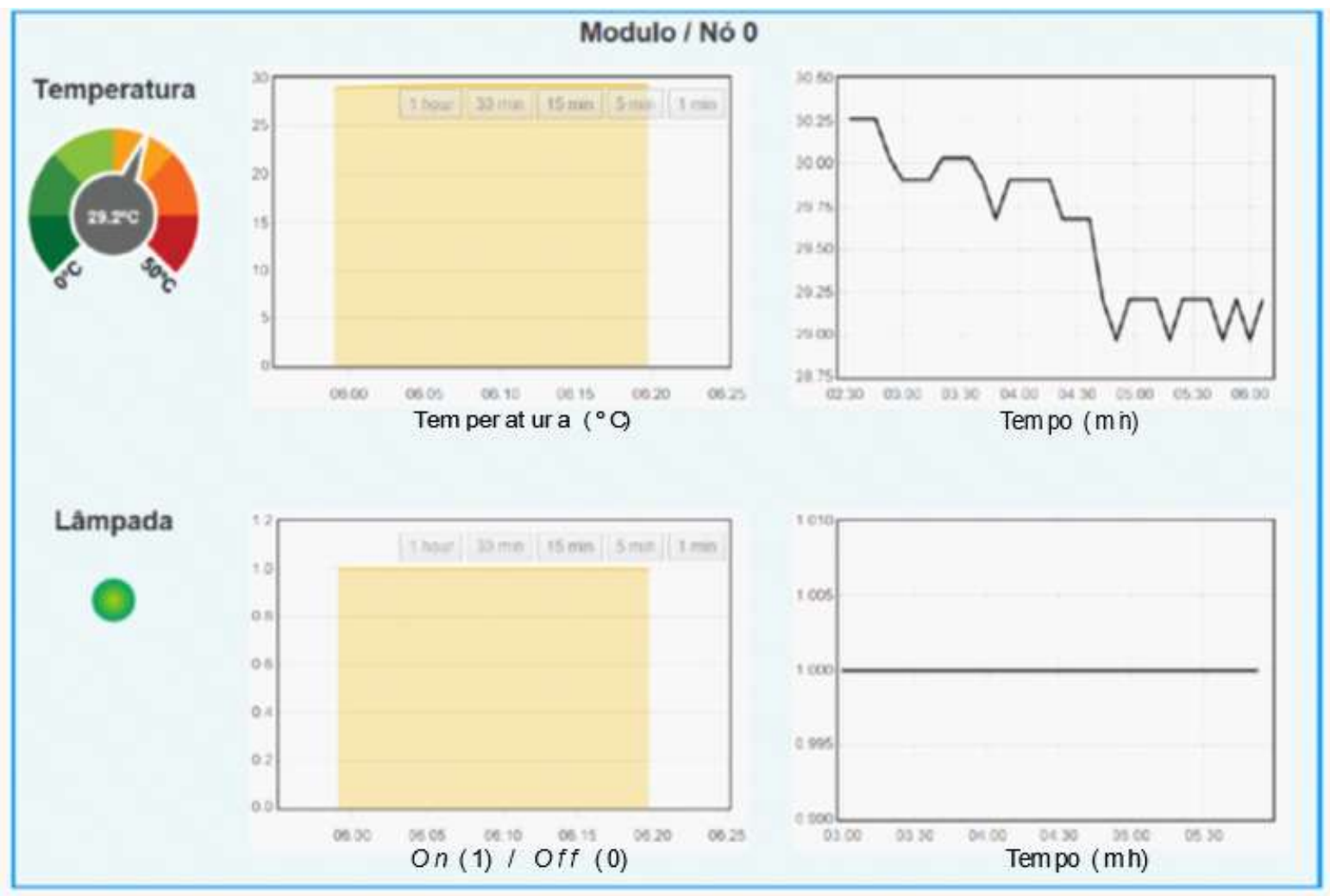

Fonte: Autores.

Quadro 1. Consumo de corrente dos componentes do dispositivo Modelo 1.

\begin{tabular}{|c|c|c|c|c|}
\hline Pin 13 XBee & LM35 & LDR & Explorer Adapter e XBee & XBee (3,3V) \\
\hline Low & $0,02 \mu \mathrm{A}$ & $0,03 \mu \mathrm{A}$ & $53,85 \mathrm{~mA}$ & $46,6 \mathrm{~mA}$ \\
\hline High & $55,83 \mu \mathrm{A}$ & $67,01 \mu \mathrm{A}$ & $17,89 \mathrm{~mA}$ & $169 \mu \mathrm{A}$ \\
\hline
\end{tabular}

Fonte: Autores.

\section{Conclusão}

O desenvolvimento de uma rede de sensores sem fio utilizando protocolo ZigBee apresentou-se eficiente para uso em ambientes comerciais e residências. O desenvolvimento dos dispositivos Coordenador e dos dispositivos Modelo 1 foram fundamentais para o funcionamento deste tipo de rede. Dessa forma, pode-se monitorar e armazenar dados por diferentes meios, permitindo alta integração entre os dispositivos de armazenamento e aplicações web. Além disso, os dispositivos finais da rede apresentaram eficiência no consumo energético, através do uso do Modo Sleep dos módulos XBee.

Para trabalhos futuros poderá ser desenvolvido uma rede Mesh com módulos ZigBee que abarcam outros tipos de sensores e de dados, como presença de seres humanos no ambiente, entre outros.

\section{Referências}

Avelino, Á. M., de Paiva Rêgo, S., de Paiva Amorim, L. G., \& Fernandes, J. D. (2012). Monitoramento de ativos hospitalares através de uma rede de sensores sem fio. Revista Brasileira de Inovação Tecnológica em Saúde.

de Melo Junior, G., de Oliveira Filho, R. M., Vieira, S. L., de Macedo, S. O., Furriel, G. P., \& Silva, B. C. R. (2020a). Rede de sensores wireless com topologia Mesh utilizando protocolo ZigBee. Research, Society and Development, 9(10), e4649108770-e4649108770.

de Melo Junior, G., de Oliveira Filho, R. M., Vieira, S. L., de Macedo, S. O., Furriel, G. P., \& Silva, B. C. R. (2020b). Desenvolvimento de uma rede de sensores wireless utilizando protocolo ZigBee para monitoramento de dados e acionamento de atuadores. Research, Society and Development, 9(10), e2729108651-e2729108651. 
da Silva, A. T. (2007). Módulos de comunicação wireless para sensores. Projecto final de curso, Universidade do Porto.

de Sousa, A. H., dos Santos, E. C., \& Curvêllo, R. (2008). Aplicação web para controle, monitoramento e parametrização de eletrodomésticos em rede sem fio. Revista do Instituto Superior Tupy, 8(9).

Farahani, S. (2011). ZigBee wireless networks and transceivers. Newnes.

Instruments, T. (1999) “LM35 Precision Centigrade Temperature Sensors”, LM35 datasheet.

Manual, P. (2008). “Xbee Znet 2.5/Xbee PRO Znet 2.5 OEM RF Modules”, Digi International Inc.

Moyolema, B. O., Silva, C. A., Casella, I. R., \& Trujillo, J. D. (2020). Otimização do posicionamento de inteligentes em redes elétricas de baixa tensão baseados em tecnologia ZigBee. Simpósio Brasileiro de Sistemas Elétricos-SBSE, 1(1).

Oliveira, T. D. A. (2015). Redes dinâmicas de sensores sem fio ZigBee para aplicações de monitoramento e controle. Universidade Estadual Paulista Júlio de Mesquita Filho.

Pechoto, M. M., Ueyama, J., \& Pereira, J. P. A. (2012). E-noé: Rede de sensores sem fio para monitorar rios urbanos. In Congresso Brasileiro Sobre Desastres Naturais.

Pereira, A. S., et al. (2018). Metodologia da pesquisa científica. UFSM. https://repositorio.ufsm.br/bitstream/handle/1/158 24/Lic_Computacao_Metodologiapesquisa-Cientifica.pdf?sequence $=1$.

Sabo, P. H., Martini, J. A., \& de Lara, R. A. (2011). Rede de sensores sem fio para monitoramento frutícola. IV EPAC - Encontro Paranaense de Computação ISSN:1981-8653

Saleiro, M., \& Ey, E. (2009). ZigBee-Uma abordagem prática. Disponível em: http://lusorobotica. com/ficheiros/Introducao_ao_Zigbee_-_por_msaleiro.pdf. Acesso em: 20/12/2020.

Silva, C. A. G., Santos, E. L., \& Ferrari, A. C. K. (2017). A study of the mesh topology in a ZigBee network for home automation applications. IEEE Latin America Transactions, 15(5), 935-942.

Sírio A. M. (2018). Comunicação de uma rede mesh utilizando o padrão ZigBee. Trabalho de Conclusão de Curso. Centro Federal de Educação Tecnológica de Minas Gerais - CEFET. Araxá.

Stuhler, J. A., Alexandrini, F., Alexandrini, C. F., De Faveri, J. E., \& Araujo, T. S. (2012). Utilização da Tecnologia Zigbee para Sensoriamento de Nível de Rio para Monitoramento de Cheias. IX SEGeT 2012 - Simpósio de excelência em Gestão e Tecnologia.

Sunrom Technologies (2008). "Light Dependent Resistor - LDR”, [S.1.].

Tavoloni Jr, P. R., Nascimento, V. E., \& Ramalho, L. A. (2013). Proposta de Metodologia de Baixo Custo para Mineração de Dados SMART GRID utilizando Redes de Sensores ZIGBEE. Sistema de Publicações. IFMT-Campus Cuiabá, 1(1).

Tose, T., Garcia, A. S., Frasson, A. M., Ramalho, L. A., \& DE N, D. (2012). Rede de Sensores sem fio Zigbee Aplicada em uma Estação de Tratamento de Esgoto. In XIX Congresso Brasileiro de Automática. CBA. 